www.nature.com/ejhg

\title{
Importance of searching for associated mitochondrial DNA alterations in patients with multiple deletions
}

\author{
R Paul $^{1}$, C Desnuelle $^{1}$, J Pouget ${ }^{2}$, JF Pellissier ${ }^{3}$, C Richelme $^{4}$, MF Monfort $^{3}$, C Butori $^{5}$, \\ A Saunieres ${ }^{1}$ and $V$ Paquis-Flucklinger ${ }^{1}$ \\ ${ }^{1}$ Laboratoire de Neurobiologie Cellulaire, UMR CNRS6549, Faculté de M édecine, Nice; ${ }^{2}$ Service de Neurologie; \\ ${ }^{3}$ Service de N europathologie, CHU Timone, Marseille; ${ }^{4}$ Service de Pédiatrie, Hôpital de I'Archet; ${ }^{5}$ Service \\ d'Anatomopathologie, Hôpital Pasteur, Nice, France
}

Multiple mitochondrial DNA (mtDNA) deletions have been reported in patients with autosomal dominant and recessive disorders. We studied several affected and one non-affected individuals belonging to a pedigree in which the inheritance of the pathological trait was compatible with an autosomique dominant transmission. Affected members had late-onset multisystem disorders with multiple mtDNA deletions in skeletal muscle. But this family presented a striking difference from previously described cases, because none of the patients had progressive external ophthalmoplegia (PEO). We also studied one young boy with a no contributary family history. He had a cerebellar ataxia with PEO and multiple mtDNA deletions in muscle. Molecular analysis revealed that in the first family, repeated sequences were present at the breakpoint junctions, whereas such motifs were not found in the young patient's case. In the first family, we evidenced mtDNA point mutations in clones containing breakpoint junctions and a 9-bp motif triplication in the intergenic COII/tRNA ${ }^{\text {Lys }}$ region, whereas this sequence is repeated twice in the wild type mtDNA. Our results suggest that multiple deletions observed in the two pedigrees result from different molecular mechanisms and point out the role of repeated sequences in the first pedigree. No mtDNA repair system has been described in mammals so far, but the molecular abnormalities found in the first family suggest that a defect in an mtDNA repair system, homologous to the E. coli MutHLS pathway, could be responsible for such a phenotype. European Journal of Human Genetics (2000) 8, 331-338.

Keywords: mitochondrial diseases; mtDNA; multiple deletions; repeated sequences; mtDNA repair system

\section{Introduction}

Mitochondrial DNA deletions have frequently been reported in patients with mitochondrial diseases. ${ }^{1,2}$ Although mtDNA is maternally inherited, single deletions are usually sporadic whereas multiple deletions are transmitted in a Mendelian fashion. All the proteins necessary for the replication and the maintenance of the mitochondrial genome are encoded by nuclear genes. Defects in such genes could result in an accumulation of de novo mtDNA deletions and could explain the Mendelian inheritance of the disease. Pedigrees with

Correspondence: Dr V Paquis-Flucklinger, Laboratoire de Neurobiologie Cellulaire, UMR CNRS6549, Faculté de Médecine, av. de Valombrose, 06107 Nice cedex 2, France. Tel: +334933777 14;

Fax: +334933777 17; E-mail: paquis@unice.fr Received 9 July 1999; revised 2 December 1999; accepted 6 January 2000 autosomal dominant progressive external ophthalmoplegia (AD-PEO) and multiple mtDNA deletions have been reported..$^{3-7}$ Given the many proteins potentially involved in the stability of the mitochondrial genome, genetic heterogeneity within AD-PEO families was predictable. This has been confirmed by linkage studies that assigned at least four different loci. ${ }^{8-10}$ But no causal gene has yet been identified in the AD-PEO families where the disease locus has been determined by linkage analysis. An interesting point concerns the existence of AD-PEO locus specific signs or symptoms. AD-PEO patients have myopathies with ptosis and progressive ophthalmoplegia. These symptoms are the major findings in the 10q-linked AD-PEO family, reported by Suomalainen et al, whereas associated signs as ataxia, deafness or cataract are mainly found in non-10q-linked AD-PEO patients. ${ }^{6}$ Nevertheless, no clinical marker is really specific 


\section{A}

\section{Pedigree 1}

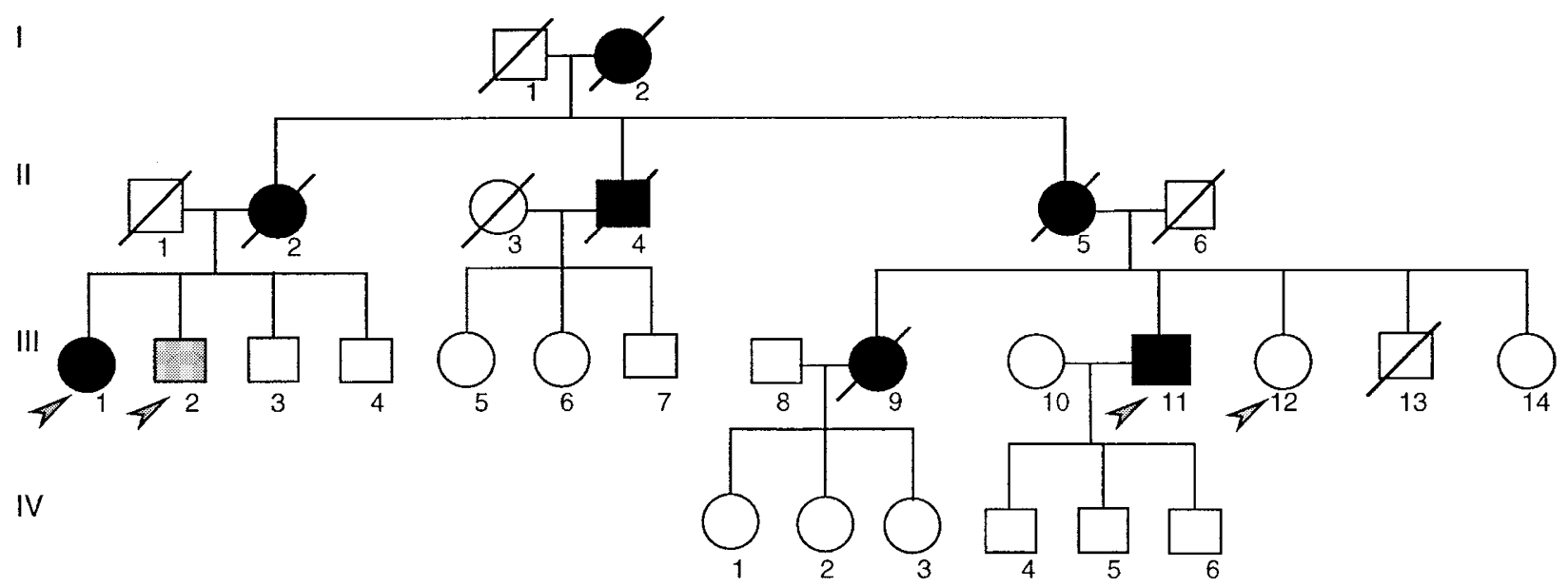

Neurological signs

Diabetes; Deafness

B

Pedigree 2

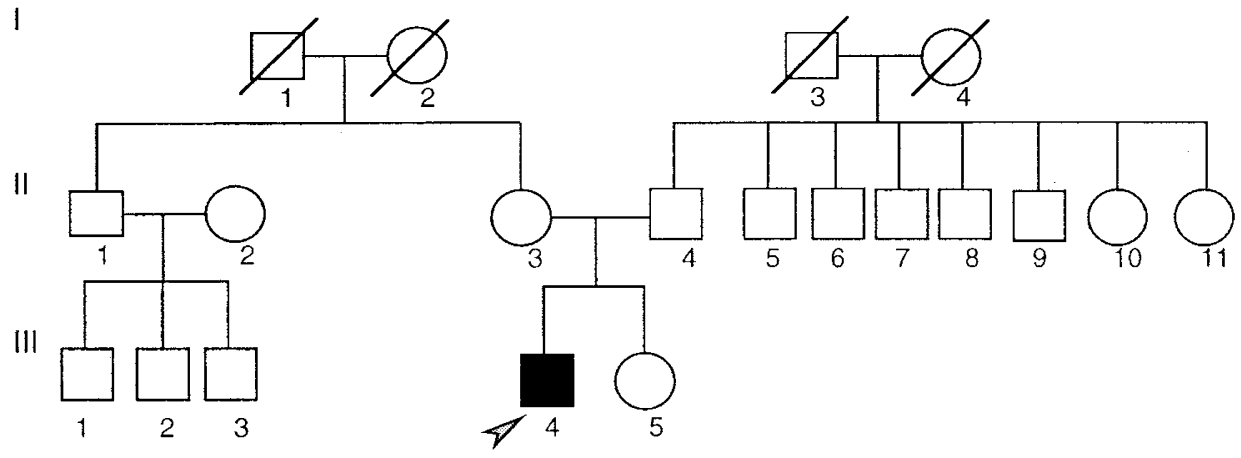

\section{Cerebellar ataxia; CPEO; Ptosis}

Figure 1 Pedigree of the families. A Family 1. B Family 2. In all cases, squares denote males and circle females. Barred symbols indicate deceased individuals. Arrows correspond to the propositi.

and this is probably explained in part by the variable distribution of deleted molecules in the different tissues. ${ }^{6,7}$ Multiple mtDNA deletions have also been described in recessive disorders including neurogastrointestinal encephalomyopathy (MNGIE) $)^{11-13}$ and cardiomyopathy with ophthalmoplegia (ARCO). ${ }^{14}$ The patients with recessive disorders have multi-organ involvement but the most striking difference from dominant forms is that they present an earlier onset. ${ }^{14,15}$

In this study, we compare three individuals from two different pedigrees, harbouring multiple mtDNA deletions in muscle. In the first family, the onset of the disease is late (5th decade) and the transmission is evocative of an autosomal dominant disorder (Figure 1A). The striking difference from 
the AD-families previously described is the absence of PEO in all affected individuals. In the second family, the disorder is evocative of a recessive form with one affected young boy only, presenting multiple mtDNA deletions in muscle (Figure1B). A progressive multi-organ involvement appeared in the course of the disease, including a PEO. Associated with a different clinical presentation, molecular findings are in favour of different mechanisms responsible for the multiple mtDNA deletions.

\section{Materials and methods Subjects}

Pedigree1 We studied two patients in a family affected by a neurological disease (Figure 1A). The detailed clinical features of the present family will be described el sewhere (Pouget et al, in preparation). Briefly, the first patient (IIII) is a 68-year-old woman. At 50 years of age, she developed a progressive ataxia, a frontal dementia and a bulbar syndrome. She had no ophthalmoplegia, ptosis or limb weakness. The second patient (IIIII) is a 63 year-old man. At 59 years of age, he first presented with dysphagia and dysarthria. Bulbar weakness, facial diplegia and shoulder muscle weakness were found at the neurological examination. Behaviour changes evoked a frontal syndrome. He had no ophthalmoplegia, ptosis, cerebellar or pyramidal signs.

In both patients, cerebral MRI was normal. EMG evidenced myogenic changes. Muscle biopsy showed numerous ragged red fibres. The histochemical reaction for cytochrome c oxidase was absent in more than $30 \%$ of the fibres. Biochemical analysis evidenced a defect in complex IV activity.

The individual III2 has a different clinical presentation with diabetes and deafness. He seems to have no neurological symptoms. Unfortunately, we have few details regarding the clinical investigations, and muscular biopsy was not performed. Individuals $12,112,114,115$ and 1119 were also affected and died of a late-onset neurological illness.

Pedigree2 PatientIII4 is an 11-year-old boy born to healthy un related parents. The family history is not contributary and he had a healthy younger sister (Figure 1B). He first presented with frequent falls and he progressively developed cerebellar ataxia. Ptosis with limitation of extraocular movements are now present. He has a mild proximal muscle weakness of the upper limbs. Tendon reflexes are absent. He has a growth failure (height and weight below -2SD) but no mental retardation, deafness, retinitis pigmentosa or cardiac involvement. Brain MRI was normal. Results of nerve conduction and electromyographic studies were consistent with neuropathy and myopathy. Histopathological examination revealed typical mitochondrial myopathy features with $30 \%$ of ragged red fibres. Biochemical analysis showed decreased activities in complexesI, III and IV.

\section{Molecular analysis}

Isolation of total DNA from frozen skeletal muscle and circulating leukocytes was performed under standard conditions. The mtDNA was amplified with the Expand Long Template PCR System (Roche, Meylan, France), as previously described. ${ }^{16}$ We used one set of primers (set 1: 1A: nt 571-598 and 1B: nt 16220-16193) to amplify in one step a $15.6 \mathrm{~kb}$ fragment, corresponding to the wild-type molecule. Southern blot analysis, with Pvu II digestion of total DNA $(5 \mu \mathrm{g})$, and slot blot procedure were conducted as previously described. ${ }^{12,17}$ Quantification of the mtDNA relative amount was performed with a $18 \mathrm{~S}$ rRNA probe as previously reported. ${ }^{12}$

The boundaries of the mtDNA deletions were amplified with several set of primers: $2 \mathrm{~A}$ : nt 8155 to nt 8176 and 2B: nt 14263 to nt 14246; 3A: nt 8128 to nt 8147 and 3B: nt 16125 to nt 16106; 4A: nt 4232 to nt 4251 and 4B: nt 9339 to nt 9320; 5A: nt 111 to nt 130 and 5B: nt 4970 to nt 4951. PCR reactions were performed under conditions depending on each primer set, with the Expand Long Template PCR System (Roche, Meylan, France). PCR products were purified using the Qiaquick PCR purification kit (Qiagen, Courtaboeuf, France) and directly cloned into the pGEM T Easy vector (Promega, Cherbonnière, France) using the single 5' A overhangs of the amplification product for the ligation. The ligated products were then used to transform Escherichia coli XL2 Blue (Stratagene, St Quentin en Yuelines, France). Double strand sequencing was performed using a T7 sequencing kit, according to the manufacturer's instructions (Pharmacia, Orsay, France).

When the 9-bp motif starting from position 8272 was present in triplicate in the intergenic COII/tRNA ${ }^{\text {Lys }}$ region, a 251 bp fragment was amplified using the set of primers 6 : nt 8155 to nt 8176 and nt 8397 to 9878 , whereas a 242 bp product was obtained from wild-type mtDNA. Amplification products were separated by electrophoresis on a $2 \%$ Metaphor agarose gel (FMC products, Rockland, ME, USA) and stained with ethidium bromide.

\section{Results \\ Identification of multiple mtDNA deletions}

Using the 'Expand Long Template' PCR method with primer set 1 , we amplified, in addition to the $15.6 \mathrm{~kb}$ wild type fragment, numerous shorter mtDNA fragments (Figure2A). This result was observed in patientsIII1, IIII1 (pedigree1) and III4 (pedigree2) when mtDNA was extracted from muscle. When mtDNA was extracted from circulating leukocytes, a unique band corresponding to the wild-type molecule was obtained. This observation, suggesting the presence of multiple mtDNA deletions in muscle, was confirmed by a Southern blot analysis (Figure2B). We found several additional bands ranging in size from 6 to $16 \mathrm{~kb}$, in addition to the $16.6 \mathrm{~kb}$ wild-type mtDNA molecules. The three patients 
A

\section{Pedigree 1}
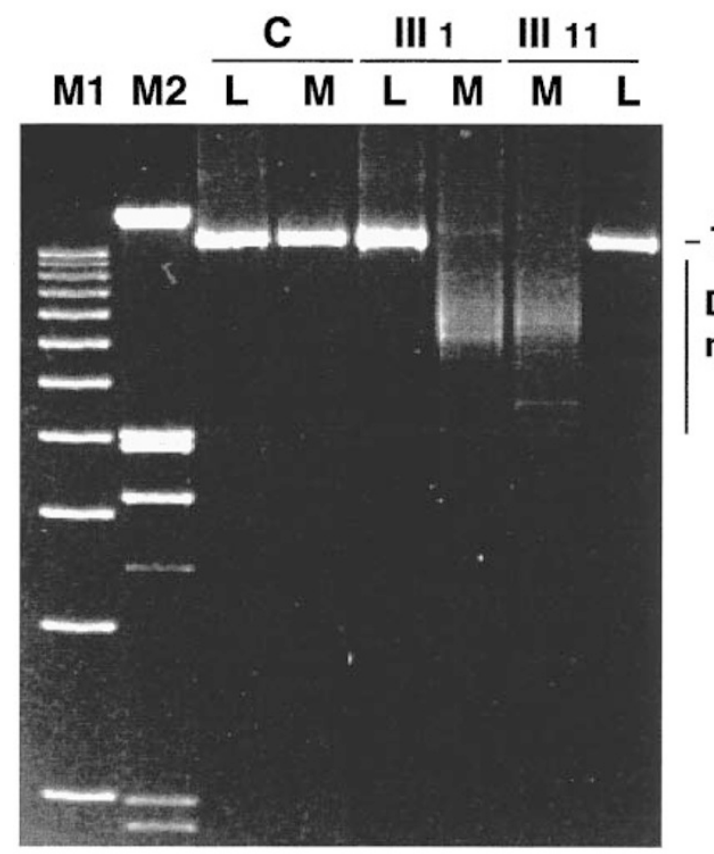

B

\section{Pedigree 1}

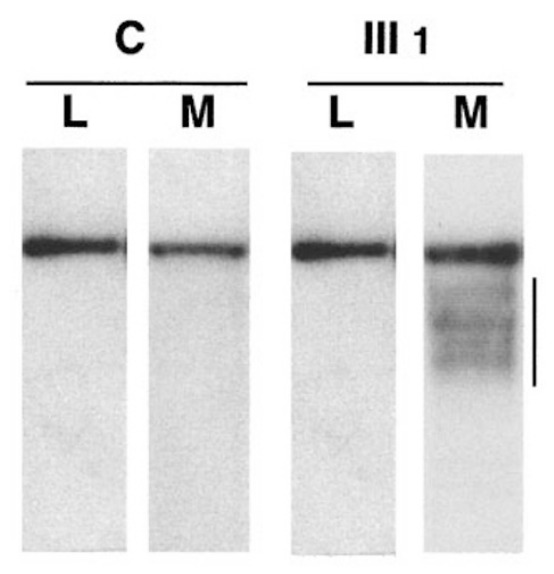

\section{Pedigree 2}

M1 M2 $\frac{C}{L} \frac{\text { III } 4}{L \quad M}$

$15.6 \mathrm{~kb}$

Deleted

molecules

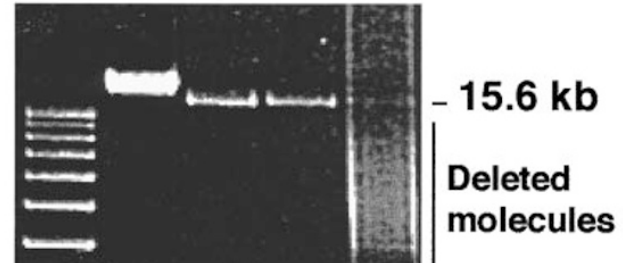

$5 \mathrm{~kb}$

2 kb

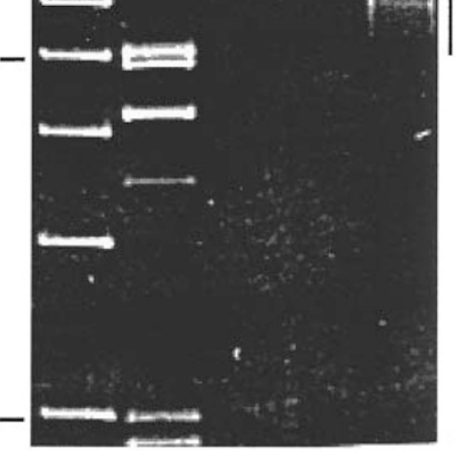

molecules

Figure 2 Mitochondrial DNA analysis. A Amplification of mtDNA by long template PCR method, using primer set 1, from a control $(C)$ and patients belonging to pedigrees 1 and 2 . The $15.6 \mathrm{~kb}$ product corresponds to the wild type molecule amplified from leukocytes (L) or muscle (M). Molecular weight markers are $1 \mathrm{~kb}$ ladder (M1) from Gibco BRL (Cergy Pontsise, France) and HindllI-EcoRI restricted $\lambda$ DNA (M2). B Hybridization pattern of Pvull-digested total DNA from leukocytes (L) or from muscle (M). The expected size of the wild-type molecule is $16.6 \mathrm{~kb}$.

and the other relatives [IIIL and III 12 (pedigree1)] who were studied had no mtDNA rearrangements in their leukocytes either by PCR or by Southern blot methods (data not shown). Papadimitriou et al reported the combination of partial depletion and multiple deletions of mtDNA in MNGIE syndrome. ${ }^{12}$ Slot blot analysis did not show mtDNA deple- tion in patients from the two pedigrees either in muscle, or in leukocytes (data not shown).

\section{PCR-based deletion mapping}

Several sets of oligonucleotides were designed in order to amplify different deletion-containing regions of mtDNA 


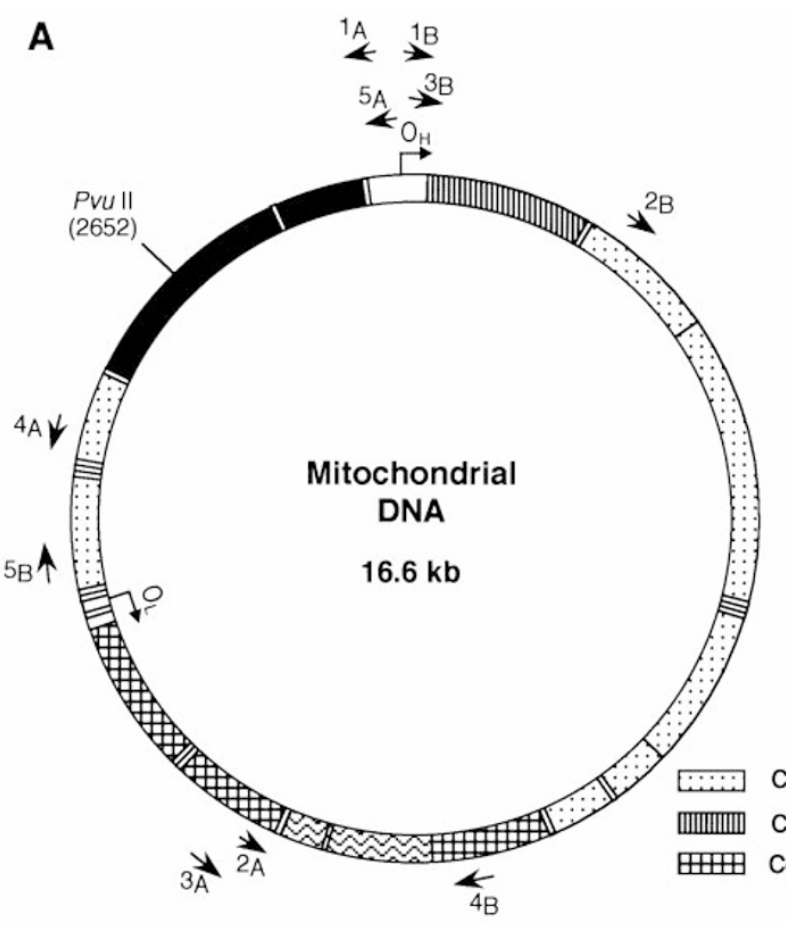

B

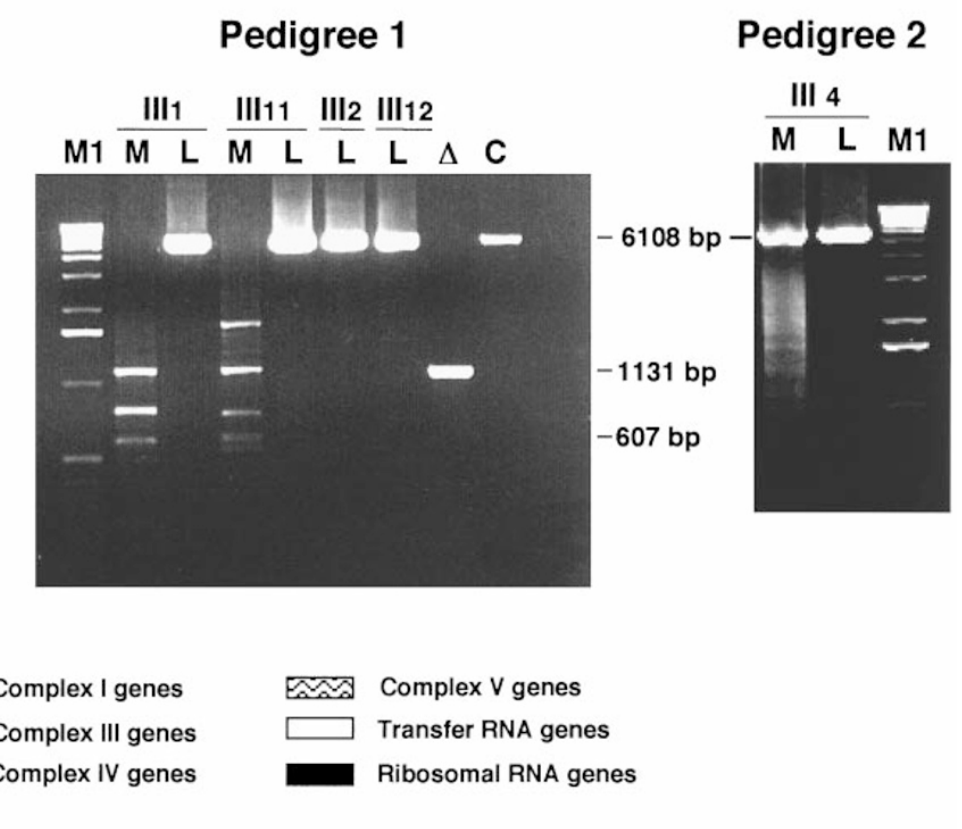

Figure 3 PCR-based deletion mapping analysis. A Schematic map of human mtDNA. Arrows show the location of different primer sets used in PCR-based deletion mapping. The unique restriction site for Pvull is also indicated. B, C PCR-based deletion mapping, using primer set 2 , in pedigrees 1 and 2 and in a control (C). The expected size of the wild type product is $6108 \mathrm{bp}$, after amplification from DNA extracted from muscle (M) or leukocytes (L). A 1131 bp fragment is preferentially amplified from a patient harbouring a unique 'common deletion' $(\Delta)$. Molecular weight marker is $1 \mathrm{~kb}$ ladder (M1) from Gibco BRL.

(Figure3A). Primer set 2 allows the amplification of a $6108 \mathrm{bp}$ wild-type fragment. When the mtDNA 'common' deletion is present, a $1131 \mathrm{bp}$ fragment, encompassing the deletion boundaries, is observed. ${ }^{18}$ We amplified the mtDNA from muscle of patientsIIII and IIIII (pedigree 1). In addition to the $1131 \mathrm{bp}$ fragment corresponding to the 'common' deletion, three to four bands were observed ranging from 0.5 to $2 \mathrm{~kb}$ (Figure3B). The same experiment was performed with patient III4 (pedigree2). Numerous bands ranging from 0.25 to more than $3 \mathrm{~kb}$ were found. No fragment corresponding in size to the 'common' deletion was clearly observed (Figure3C).

With a reverse primer located in the D-loop region (set 3), numerous bands ranging from $0.5 \mathrm{~kb}$ to $3 \mathrm{~kb}$ were observed in muscle of the three patients in addition to the $7997 \mathrm{bp}$ wildtype fragment (data not shown).

Always in addition to the wild-type fragment (5107 bp), primer set 4 amplified one band of $1 \mathrm{~kb}$ and two bands of 1 and $4 \mathrm{~kb}$ from muscle of patientsIIII and III 11 respectively (pedigree1). Primer set 4 failed to amplify any fragment corresponding to a del eted molecule, from muscle of patientIII4 (pedigree2) (data not shown).

Primer set 5 allows the amplification of a 4859 bp wild-type fragment. A 965 bp band is observed when the 'HSP' deletion is present. This deletion is located near the heavy-strand promoter and has been previously reported by M oraes et al. ${ }^{19}$
The $965 \mathrm{bp}$ band with others up to $2 \mathrm{~kb}$ were obtained from muscle of patientsIIII and IIIII (pedigree1). Primer set 5 failed to amplify a fragment corresponding to the ' $\mathrm{HSP}$ ' deletion, from muscle of patient III4 (pedigree2). But many deleted molecules were present (data not shown).

Amplifications with primer sets 1 to 5 failed to identify deleted molecules from leukocytes of the three patients.

\section{The characterisation of mtDNA deletion breakpoints reveals the presence of repeat motifs in pedigree 1 but not in pedigree 2}

PCR fragments, amplified from the three patients with primer sets 2 and 3, were cloned and sequenced. Within the mtDNA of patientsIIII and IIIII (pedigree1), we characterised 6 and 5 different deletion breakpoints respectively (Table1). With the same primer sets, 10 boundaries were identified for patientIII4 (pedigree2). Sequence analysis of the breakpoint regions revealed that repeated sequences were al ways found at the breakpoint junctions with in the mtDNA of patientsIIII and IIIII (pedigree1). In contrast, such sequences were rarely found for the patient III4 (pedigree2).

\section{The sequencing of clones containing the breakpoint regions reveals different abnormalities in pedigree 1}

The inserts present in clones containing the breakpoint regions amplified with primer set 2 were entirely sequenced 
Table 1 Nucleotide sequences of breakpoint regions amplified with primer sets 2 and 3. Breakpoints are indicated by parentheses and italic letters indicate nucleotides within deletions. Numbers refer to nucleotide positions in the Cambridge sequence on human mtDNA L strand. Regions of homology at each side of the deletions are underlined. Direct repeats are single underlined and indirect repeats are double underlined. Sequences with no complete homology are dotted underlined. Arrows indicate inverted repeats. The number of each clone (\#) and the size of the inserts are also included

\begin{tabular}{|c|c|}
\hline Patient III 1 & Patient III 4 \\
\hline Primer set 2 & Primer set 2 \\
\hline $\begin{array}{lcc}\text { \#2 } & 8266 & 13928 \\
\text { 447bp GG CCC GTA TTT ACC CTA } & (\ldots \text {...TC TAC CCTA) GC ATC AC }\end{array}$ & 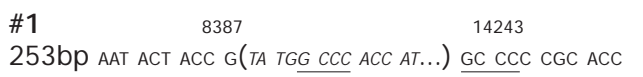 \\
\hline $\begin{array}{lcc}\# 7 & 8643 & 14216 \\
536 \mathrm{bp} \text { CTC ATC AAC (AAC CGA CTA ATCA...) } & \text { AC TAA TCA ACG }\end{array}$ & $\begin{array}{lcc}\# 2 & 8546 & 14243 \\
412 \text { bp CTG TTC GC(T TCA TTC ATT G... ...ATA CAA A)GC CCC CGC }\end{array}$ \\
\hline $\begin{array}{lcc}\# 11 & 8289 & 13791 \\
607 b p \text { cCTCTA } & \text { CCCCCTCTA }(\ldots \ldots . . \text { ATC CCC CTC TA }) & \text { C CTA AAA }\end{array}$ & $\begin{array}{lcc}\# 3 & 8487 & 14243 \\
263 b p \text { TCA CCA AAG CC(C ATA... ....ATA CAA A) GC CCC CGC AC }\end{array}$ \\
\hline $\begin{array}{lrr}\# 23 & 8482 & 13460 \\
1131 \text { bp с CTA CCT CCC TCA CCA }(\ldots . \text { ACC TCC CTC ACCA }) & \text { TTGGC }\end{array}$ & $\begin{array}{lcc}\# 4 & 8286 & 14225 \\
169 b p \text { TCTA CCCCCT (CTAG AGCC... ....CTA ATC AA) C GCC CAT A }\end{array}$ \\
\hline Primer set 3 & Primer set 3 \\
\hline 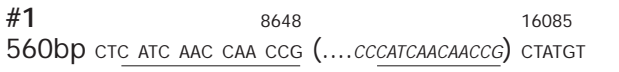 & $\begin{array}{lcc}\# 2 & 8278 & 16034 \\
\text { 238bp cACCCCCCTC(TACCCCCCTCT.. ...TCTTTCATG)GGGAAGCAGA }\end{array}$ \\
\hline $\begin{array}{lcc}\# 4 & 8397 & 16070 \\
321 \text { bp ACC GTA TGG CCC AC (...AAGTATTGACTC) } & \text { ACCCATCAAC }\end{array}$ & $\begin{array}{lcc}\# 4 & 8305 & 15948 \\
354 \text { bp ACTGTAAAGC }(\text { TAACTTAGCA... ....CTTTTTCCA)AGGACAAAT }\end{array}$ \\
\hline Patient III 11 & $\begin{array}{lcc}\# 6 & 8208 & 16047 \\
155 b p & \text { TTC ATG CC(C ATC GTC CTA... ... AAGCAGATTT)GGGTACCA }\end{array}$ \\
\hline Primer set 2 & $\begin{array}{lll}\# 7 & 8375 & 16035\end{array}$ \\
\hline $\begin{array}{l}\text { \#3 } \\
534 \text { bp cC CAA CTA AAT ACT ACCG (...CAACTACCTAACCA) ACAA }\end{array}$ & $\begin{array}{l}\text { 334bp ATG CCC CAA C(TA AAT ACT....... } \\
\# \mathbf{8}\end{array}$ \\
\hline $\begin{array}{lcc}\# 4 & 8278 & 14070 \\
317 \text { bp TAGCACCCCCTC } & (\ldots \ldots \ldots \ldots . . . . . . A T C A C C T C) & \text { AACCCAAAAAG }\end{array}$ & 317bp ACC GT (A TGG CCC ACCAT... ..GGTACCACCCA AGTAT)TGAC \\
\hline 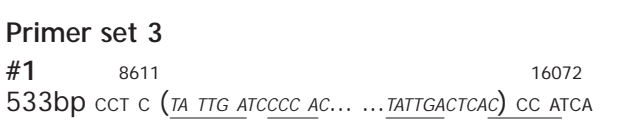 & 335bp CCC CAA C(TA AAT ACT ACC.......TTCTTTCATG)GGGAAGCA \\
\hline $\begin{array}{lll}\# 2 & 8387 & 16069 \\
312 \text { bp ACC G (TA TGG CCC ACC... ....AGTATTGACT) } & \text { CACCCATCA }\end{array}$ & \\
\hline $\begin{array}{lcc}\# 5 & 8276 & 16074 \\
196 b p \text { AG CACCCCCC (TCTACCC.......ATTGACTCACCC) } & \text { ATCAAC }\end{array}$ & \\
\hline
\end{tabular}

on both strands. In patientsIIII and IIIII (pedigree1), two previously described polymorphisms (G14167T and T14199C) were found in all corresponding clones. ${ }^{20,21}$ In patient IIII (pedigree1), point mutations were found in three clones out of four. One point mutation was present in clone11, two in clone2 and four in clone23 (Table2). In patient IIII1 (pedigree 1), two clones out of two contained a point mutation. In patient III4 (pedigree2), only a A8440G mutation was found in one clone out of four.

In the wild-type human mtDNA, a 9-bp motif (CCCCCTCTA) is repeated twice at positions 8272 to 8280 and 8281 to 8290 in the intergenic COII/tRNA ${ }^{\text {Lys }}$ region. One copy of the same motif is found at positions 13781 to 13790 . This sequence is involved in one of the deletions (corresponding to clone11) observed in patient III1. Furthermore, we have found that this motif is present in triplicate from position 8272 in both patients of pedigree1 (Figure4). We looked for the presence of this motif in triplicate, from position 8272, in patients from the 2 pedigrees and in controls (Figure4). It was observed in a homoplasmic state in blood and muscle of patient III11 (pedigree1). It was present in a heteroplasmic state in blood and muscle of patient III 1

Table 2 Nucleotide transitions observed in deletioncontaining regions amplified with primer set 2

\begin{tabular}{|c|c|c|c|c|}
\hline Patients & $\begin{array}{l}\text { Clone } \\
\text { number }\end{array}$ & Gene & $\begin{array}{l}\text { Nucleotide } \\
\text { transition }\end{array}$ & $\begin{array}{l}\text { Amino-acid } \\
\text { remplacement }\end{array}$ \\
\hline \multicolumn{5}{|l|}{ Pedigree 1} \\
\hline \multirow[t]{3}{*}{ Patient IIII } & 2 & $\begin{array}{l}\text { ND5 } \\
\text { ND5 }\end{array}$ & $\begin{array}{l}\text { T13961C } \\
\text { T14146C }\end{array}$ & $\begin{array}{l}\text { Leu->Phe } \\
\text { Stop->GIn }\end{array}$ \\
\hline & 11 & ND5 & C13798T & Leu->Phe \\
\hline & 23 & $\begin{array}{l}\text { tRNAlys } \\
\text { ND5 } \\
\text { ND6 } \\
\text { ND6 }\end{array}$ & $\begin{array}{l}\text { T8357C } \\
\text { A14027G } \\
\text { C14239T } \\
\text { T14218C }\end{array}$ & $\begin{array}{l}\text { Lys->->ys } \\
\text { Leu-> - Leu } \\
\text { Leu-> Leu }\end{array}$ \\
\hline \multirow[t]{2}{*}{ Patient III11 } & 3 & ND5 & A14014G & Lys->Glu \\
\hline & 4 & ND6 & T14221C & Trp->Trp \\
\hline \multicolumn{5}{|l|}{ Pedigree 2} \\
\hline Patient III4 & 2 & ATPase8 & A8440G & $\mathrm{Gln}->\mathrm{Gln}$ \\
\hline
\end{tabular}




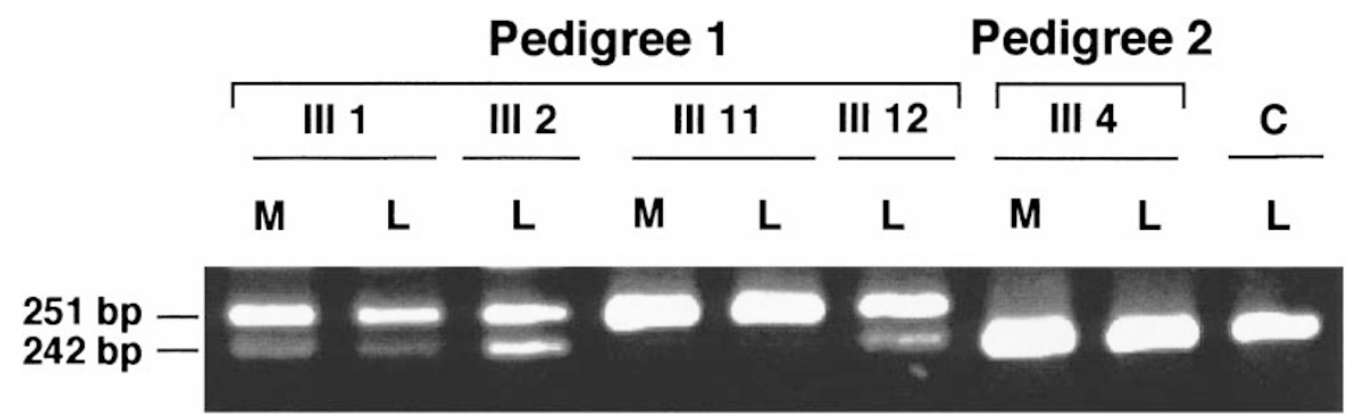

Figure 4 PCR analysis of the intergenic COII/tRNA ${ }^{\text {Lys }}$ region in pedigrees 1,2 and in a control (C). The expected size of the wild-type PCR product is $242 \mathrm{bp}$. Amplifications were performed from DNA extracted from muscle (M) or leukocytes (L).

and in blood of the other relatives (III2 and III12 [pedigree1]). We never found three copies of this motif from position 8272 in patient III4 (pedigree2), or in 50 controls.

\section{Discussion}

In this report, we compare two different families with affected individuals harbouring multiple mtDNA deletions in muscle. The mode of transmission, the age of onset, the clinical history are different. In the first family, the deletions appear to be inherited in a M endelian manner, being possibly dominant unlike the absence of a father's transmission. A striking difference from the previously reported AD-families harbouring mtDNA deletions is the absence of ptosis and ophthalmoplegia in the patients. In the second family, the affected individual was much younger at onset, a common feature in autosomal recessive disorders. All affected individuals belonging to the two pedigrees evidenced typical morphologic and biochemical abnormalities of mitochondrial disorders in skeletal muscle. In a recent study, Carozzo et al found low numbers of abnormal muscle fibres in AR-PEO patients. ${ }^{15}$ In contrast, $30 \%$ of ragged red fibres were found in the muscle of patientIII4 (pedigree2), atypical for his young age. All these differences illustrate the difficulties of searching for responsible genes on the basis of clinical features.

Molecular analysis of the mitochondrial genome showed several differences among patients belonging to the two families. Repeated sequences were present at the breakpoint junctions in the first pedigree. Such motifs were not found in the young patient's case indicating that the involvement of repeated motifs in deletional mechanism was probably not essential in the second pedigree. In their comparative study, Carozzo et al found the HSP deletion in the AD-PEO but not in the AR-PEO patients. ${ }^{15}$ We found this deletion in patientSIIII and IIIII from pedigree 1 , after cloning and sequencing the PCR products resulting from an amplification with the oligonucleotide set5 (data not shown). Using the same method we did not find the HSP deletion in patient III4 from pedigree2, among sequenced clones that were all different and did not contain repeat motifs (data not shown). Carozzo et al suggested that the presence of the HSP deletion might be a 'genetic marker' of the AD-PEO disorders. The breakpoint junctions of this deletion is flanked by obvious repeated sequences. In that instance the HSP deletion was not found in a AD-PEO Finnish family, ${ }^{6}$ linked to chromosome10. In this family, mtDNA multiple deletions were not flanked by repeated sequences. Thus, in our opinion, the HSP deletion might be a marker of a deletion mechanism involving repeated motifs rather than a marker of AD-PEO disorders.

The complete sequencing of the inserts, resulting from the amplification with primer set 2 , highlighted an interesting point. In the first pedigree, it revealed the presence of clonespecific point mutations. The T13961C mutation found in clone2 (patient IIII) creates a Mnll restriction site. PCR-RFLP analysis was performed to estimate the percentage of mtDNA molecules harbouring this mutation in this patient. We did not observe digested PCR fragments resulting from the creation of a new Mnl1 restriction site (data not shown). It is difficult to ascertain the significance of the point mutations found in pedigree1. One cannot exclude errors of polymerases but the amplification was performed with an enzyme mix containing Taq and Pwo DNA polymerases, with an error rate of $15.10^{-6}$ (Expand Long template PCR system, Boehringer). On the other hand, all the 11 deletion breakpoints characterised in pedigree 1 were different. Presumably the percentage of each mutation is too low to detect easily whether a common mechanism generates a combination of de novo deletions and point mutations. Our results need to be confirmed by further studies in other families.

The intergenic region between the cytochrome oxydasell and lysine tRNA genes (COII/tRNALys) contains two tandemly repeated copies of the 9-bp sequence CCCCСТCTA. The triplication of this 9-bp motif seems to berare. ${ }^{22,23}$ In the first family, the CССССТСТA sequence is involved both in a large deletion (patientIII1, clone11) and in the expansion of the repeat observed in the four individuals tested. One of them is asymptomatic (III12) but all had an affected mother (II2 and II5). The presence of the triplication in homoplasmic and heteroplasmic states in the same generation is not in favour of a polymorphism. A relationship between replication slippage of repeated sequences and a defect in a DNA repair system has been advanced. ${ }^{24}$ In the first pedigree, deletions and the 9-bp triplication might be secondary to 
unrepaired errors resulting from slipped mispairing during replication.

No post replicative mtDNA repair system gene has been yet identified in mammals. In E. coli, the MutS protein belongs to the MutHLS mismatch repair system. A mutation in the muts gene results in an accumulation of point mutations as well as in deletions, increased by the presence of repeated motifs. ${ }^{25}$ In Saccharomyces cerevisiae, the MSH 1 (MutS Homolog 1) gene encodes for a mitochondrial protein. ${ }^{26,27}$ MSH1-deficient yeast mutants present a 'petit' phenotype, characteristic of a functional deficiency of the respiratory chain, with mtDNA point mutations and major rearrangements. The mtDNA alterations found in the first family reported in our study might point to a defect in a human homolog of the Saccharomyces cerevisiae MSH 1 gene. Further studies will be necessary to determine whether a defect in a mtDNA mismatch repair system is involved in some families with multiple deletions. However, a careful molecular analysis can help to classify these disorders in the aim to facilitate linkage analyses and identification of candidate genes.

\section{Acknowledgements}

We thank Catherine Guerin for expert technical help, Georges Carle, Frederique Vidal and Jean Claude Scimeca for reading the manuscript and Dr Attarian for hel pful discussion. This work was made possible by grants from the Association Française contre les Myopathies (AFM).

\section{References}

1 DiMauro S, Bonilla E, Davidson M, Hirano M, Schon EA: Mitochondria in neuromuscular disorders. Biochim Biophys Acta 1998; 1366: 199-210.

2 Zeviani M, Tiranti V,Piantadosi C: Mitochondrial disorders. Medicine (Baltimore) 1998; 77: 59-72.

3 Zeviani M, Servidei S, Gellera C, Bertini E, DiMauro S, DiDonato S: An autosomal dominant disorder with multiple deletions of mitochondrial DNA starting at the D-loop region. Nature 1989; 339: 309-311.

4 Zeviani M, Bresolin N, Gellera C et al: Nucleus-driven multiple large-scale deletions of the human mitochondrial genome: a new autosomal dominant disease. Am J Hum Genet 1990; 47: 904-914.

5 Servidei S, Zeviani M, Manfredi $G$ et al: Dominantly inherited mitochondrial myopathy with multiple deletions of mitochondrial DNA: clinical, morphologic, and biochemical studies. Neurology 1991; 41: 1053-1059.

6 Suomalainen A, Majander A, Wallin M et al: Autosomal dominant progressive external ophthalmoplegia with multiple deletions of mtDNA: clinical, biochemical, and molecular genetic features of the 10q-linked disease. Neurology 1997; 48: 1244-1253.

7 Moslemi AR, Melberg A, Holme E, Oldfors A: Autosomal dominant progressive externe ophthal moplegia: Distribution of multiple mitochondrial DNA deletions. Neurology 1999; 53: 79-84.

8 Suomalainen A, Kaukonen J, Amati $P$ et al: An autosomal locus predisposing to deletions of mitochondrial DNA. Nat Genet 1995; 9: $146-151$.

9 Kaukonen JA, Amati P, Suomalainen A et al: An autosomal locus predisposing to multiple deletions of mtDNA on chromosome3p. Am J Hum Genet 1996; 58: 763-769.
10 Kaukonen J, Zeviani M, Comi GP, Piscaglia MG, Peltonen L, Suomalainen A: A third locus predisposing to multiple deletions of mtDNA in autosomal dominant progressive external ophthalmoplegia. Am J Hum Genet 1999; 65: 256-261.

11 Hirano M, Silvestri G, Blake DM et al: Mitochondrial neurogastrointestinal encephalomyopathy (M NGIE): clinical, biochemical, and genetic features of an autosomal recessive mitochondrial disorder. Neurology 1994; 44: 721-727.

12 Papadimitriou A, Comi GP, Hadjigeorgiou GM et al: Partial depletion and multiple deletions of muscle mtDNA in familial MNGIE syndrome. Neurology 1998; 51: 1086-1092.

13 Nishino I, Spinazzola A, Hirano M: Thymidine phosphorylase gene mutations in MNGIE, a human mitochondrial disorder. Science 1999; 283: 689-692.

14 Bohlega S, Tanji K, Santorelli FM, Hirano M, Al JA, DiMauro S: Multiple mitochondrial DNA deletions associated with autosomal recessive ophthalmoplegia and severe cardiomyopathy. Neurology 1996; 46: 1329-1334.

15 Carrozzo R, Hirano M, Fromenty $B$ et al: Multiple mtDNA deletions features in autosomal dominant and recessive diseases suggest distinct pathogeneses. Neurology 1998; 50: 99-106.

16 Paul R, Santucci S, Saunieres A, Desnuelle C, Paquis-Flucklinger V: Rapid mapping of mitochondrial DNA deletions by large-fragment PCR. Trends Genet 1996; 12: 131-132.

17 Maniatis T, Fritsch EF, Sambrook J: Molecular Cloning: A Laboratory Manual, 2nd edn. Cold Spring Harbor Laboratory Press: Cold Spring Harbor, NY, 1989.

18 Schon EA, Rizzuto R, Moraes CT, Nakase H, Zeviani M, DiMauro S: A direct repeat is a hotspot for large-scale deletion of human mitochondrial DNA. Science 1989; 244: 346-349.

19 Moraes CT, Andreetta F, Bonilla E, Shanske S, DiMauro S, Schon EA: Replication-competent human mitochondrial DNA lacking the heavy-strand promoter region. Mol Cell Biol 1991; 11: 1631-1637.

20 Blanc $\mathrm{H}$, Chen $\mathrm{KH}, \mathrm{D}^{\prime}$ Amore MA, Wallace DC: Amino acid change associated with the major polymorphic Hincll site of Oriental and Caucasian mitochondrial DNAs. Am J Hum Genet 1983; 35: 167-176.

21 Howell N, Kubacka I, Halvorson S, Howell B, McCullough DA, Mackey D: Phylogenetic analysis of the mitochondrial genomes from Leber hereditary optic neuropathy pedigrees. Genetics 1995; 140: 285-302.

22 Fabrizi GM, Tiranti V, Mariotti $C$ et al: Sequence analysis of mitochondrial DNA in a new maternally inherited encephalomyopathy. J Neurol 1995; 242: 490-496.

23 Alves SJ, Guimares PE, Rocha J, Pena SD, Prado VF: Identification in Portugal and Brazil of a mtDNA Lineage Containing a 9-bp Triplication of the Intergenic COII/tRNALys Region. Hum Hered 1999; 49: 56-58.

24 Strand M, Prolla TA, Liskay RM, Petes TD: Destabilization of tracts of simple repetitive DNA in yeast by mutations affecting DNA mismatch repair. Nature 1993; 365: 274-276.

25 Kolodner R: Biochemistry and genetics of eukaryotic mismatch repair. Genes Dev 1996; 10: 1433-1442.

26 Reenan RA, Kolodner RD: Isolation and characterization of two Saccharomyces cerevisiae genes encoding homologs of the bacterial HexA and MutS mismatch repair proteins. Genetics 1992; 132: 963-973.

27 Reenan RA, Kolodner RD: Characterization of insertion mutations in the Saccharomyces cerevisiae MSH 1 and MSH2 genes: evidence for separate mitochondrial and nuclear functions. Genetics 1992; 132: $975-985$. 